\title{
Special Projects of National Significance Systems Linkages Initiative (2011 to 2016): Improving Access to Care for Hard-to-Reach Populations Living with HIV
}

\author{
Janet J. Myers ${ }^{1}$ - Jessica M. Xavier ${ }^{2}$ \\ Published online: 18 December 2018 \\ (c) Springer Science+Business Media, LLC, part of Springer Nature 2018
}

The National HIV/AIDS Strategy (NHAS [1]), first released in July 2010, had three primary goals: reducing HIV infections, increasing access to care and optimizing health outcomes for people living with HIV (PLWH) and reducing HIV-related health disparities. The NHAS recognized the importance of getting people with HIV into care early after infection to protect both their health and to reduce the potential that they could transmit the virus to others. To accomplish these inter-related goals, the U.S. Department of Health and Human Services recognized the urgent need to better coordinate public health system linkages to counseling and testing, surveillance, referrals, and care provision among a more diverse group of traditional HIV-focused and newer non-traditional partners. HIV disproportionately affects people who, because of issues of stigma, discrimination and health inequity, often have less access to prevention and treatment services and, as a result, often have poorer health outcomes. For these reasons, the NHAS advocated for the adoption of community-level approaches to reduce HIV infection in vulnerable communities.

The NHAS Updated to 2020 [2] released in July 2015 added a fourth goal: achieving a more coordinated national response to the HIV epidemic. Towards that end, the NHAS Updated to 2020 required the Federal government and state, tribal and local governments to increase collaboration, efficiency, and innovation. Along with other federal agencies, NHAS 2020 tasked the HIV/AIDS Bureau (HAB) of the Health Resources and Services Administration (HRSA) with collaborating with states and localities on pilot initiatives for expanding the most promising models for integrating HIV testing, outreach, linkage and retention in high-risk

\footnotetext{
$\triangle$ Janet J. Myers

Janet.Myers@ucsf.edu

University of California, San Francisco, USA

2 Independent Consultant, Silver Spring, MD, USA
}

communities. It also called upon HRSA/HAB to develop plans to support health care providers and other staff who deliver HIV test results to develop methods to increase linkage to ensure clients receive appropriate care following an HIV diagnosis, and to work with states, tribal governments, localities, and community-based organizations (CBOs) to promote co-location of providers of HIV screening and care services as a means of facilitating linkages to care and treatment, and to enhance current referral systems within CBOs.

In anticipation of these emerging programmatic emphases, in 2011, HRSA/HAB, through its Special Projects of National Significance (SPNS) Program, funded six demonstration states and an Evaluation and Technical Assistance Center (ETAC) under a new initiative, Systems Linkages and Access to Care for Populations at High Risk of HIV Infection (hereafter, the Systems Linkages Initiative). The demonstration states (Massachusetts, New York, Virginia, North Carolina, Louisiana and Wisconsin) were all grant recipients of record funded under the Ryan White HIV/AIDS Program (RWHAP) Part B grants to states and territories. States were tasked with the development and implementation of innovative systemic models of linkage to improve access to and retention in quality HIV care for the hardest-to-reach populations living with HIV. These populations included those PLWH but unaware of their HIV status; PLWH aware of their HIV infection but who were never referred to care; PLWH aware of their HIV infection but who refused referral to care; and PLWH who have dropped out of care. The funded states were expected to leverage their SPNS funding with existing resources to work with key partners and stakeholders across their state's public health system and communities to promote the development and implementation of innovative strategies that successfully integrated different components of the public health system, such as surveillance and counseling and testing. States were required to participate in a five-year comprehensive multi-state evaluation led by a central evaluation center at the University of California, 
San Francisco's Center for AIDS Prevention Studies (UCSF CAPS) to identify and document successful models for purposes of dissemination and replication at the national level.

The initiative defined systems linkage as the enhancement of existing, or the implementation of new sustainable collaborative relationships or partnerships among RWHAP-funded and other, non-traditional HIV service organizations, such as Community Health Centers. Previous RWHAP cross-part collaborative initiatives had shown that effective systems linkage can result in optimal HIV program coordination, as demonstrated by improvements in the provision of care to PLWH. Thus, states were called upon to establish effective and sustainable systemic linkages to optimize the coordination of existing HIV counseling and testing, surveillance, prevention and treatment resources within their jurisdictions. In addition to other RWHAP-funded organizations co-located in their states, SPNS demonstration states were expected to establish linkages with traditional and non-traditional HIV service partners such as hospital emergency rooms; community health centers; outpatient, urgent care, sexually transmitted disease, mental health and/or substance abuse treatment clinics; correctional health-care facilities; and other primary care settings.

In this special supplemental issue of AIDS and Behavior, we present a collection of articles representing findings, best practices and lessons learned through the SPNS Systems Linkages Initiative. HIV patient navigation, now a ubiquitous intervention to improve linkage to and retention in HIV primary care, was first pioneered during the SPNS Outreach Initiative (2001-2006) [3]. Schumann et al. [4] describe a patient navigation intervention targeting PLWH who were out of HIV care or at risk of falling out of care. The intervention provided intensive case management and care coordination services over a nine-month period to clients, helping them identify and then overcome barriers to engaging in HIV care and attaining viral suppression. Next, Fuller et al. [5] provide a qualitative study of the experiences of participants enrolled in patient navigation interventions in four of the demonstration states focusing on the improvement of HIV care continuity for PLWH newly released from prisons. In addition to health system navigation, participants also received case management and social support. Their findings emphasize the value of patient navigation in assuring continuity of care and reintegration for PLWH upon release from prison.

Several demonstration states focused on the needs of PLWH who were newly released from incarceration. Bailey et al. [6] describe the development, implementation and outcomes of a Care Coordination Program (CCP) for newly released incarcerated persons living with HIV from Virginia prisons and jails. The intervention facilitated medication access and immediate linkage to community care providers upon release. The authors describe taking a relationships-first approach which entailed the building of collaborative relationships with the Virginia Department of Corrections and community partners. Outcomes from the CCP included 95\% linkage to HIV care within 90 days of release; and by 12 months following their release $97 \%$ had evidence of HIV care; 94\% were retained in care, and 68\% were virally suppressed. Next Brantley et al. [7] describe a video conference intervention added as a component to HIV pre-release services provided in Louisiana's adult state prisons. Prior to their release from incarceration, PLWH were offered a video conference with a RWHAP-funded case manager. The video conference session afforded PLWH an opportunity to discuss their plans for returning to their communities, continuing their HIV care, and how RWHAPfunded services could best support their needs. Although there was little difference in rates of linkage between comparison and study groups, clients and providers alike appreciated having access to the video conference supplement.

Several interventions employed social network strategies [8] to identify the target populations of the initiative: those who were PLWH but unaware of their HIV status; PLWH aware of their HIV infection but who were never referred to care; PLWH aware of their HIV infection but who refused referral to care; and PLWH who have dropped out of care. Schumann and colleagues [9] present a mixed methods evaluation of a non-clinical HIV testing program implemented by six agencies in Wisconsin using a social network recruitment strategy. The program targeted high-risk individuals in areas with lower overall HIV prevalence, and the authors describe program outcomes and some important lessons learned, including careful selection of agencies to avoid oversaturation of testing areas; close collaboration and communication between participating agencies; careful selection and supervision of recruiters; and prudent management of incentives. Next, LeViere et al. [10] present a clinic-based testing initiative utilizing social networks to increase their positive screens up to $5 \%$. The intervention went beyond just partner testing by providing free HIV testing to anyone who accompanied an HIV-infected patient to the two participating clinics.

Fadul et al. [11] compare patient outcomes from two of the four interventions fielded by North Carolina focusing on retention and re-engagement. Their clinic-based retention intervention used CAREWare, a free, electronic health and social support services information system for Ryan White HIV/AIDS Program grant recipients and their providers, to identify clients who had not attended medical appointments in the previous 6 to 9 months and did not have a scheduled appointment. They then followed a protocol to search for and re-engage these clients. When those clients could not be found, they were referred to State Bridge Counselors (SBCs) a statewide public health department team assigned to regions in the state. The SBCs worked to locate 
and re-engage those patients who were out of care for 6 to 9 months or longer, and used strengths-based counseling techniques to increase self-efficacy, eliminate barriers, and facilitate patient efforts to re-establish medical care. Clients referred to the SBC group were less likely to have viral load tests at their last visit prior to referral and were less likely to re-engage in care within 180 days, compared to clients who received clinic-based retention services only. In a qualitative study, Parnell et al. describe the encounters between PLWH who were out of care and their State Bridge Counselors focused on re-engagement in care [12].

The CDC's Data To Care initiative [13] has spurred the use of HIV surveillance data as a means of identifying those who are diagnosed and in need of being linked to care. Increasingly such efforts have become inclusive of re-engaging those who have may fallen out of care [14]. Zamudio-Haas et al. present a qualitative examination of the efforts of three states to "close the loop" and bring routinely reported patient information back to care providers to improve tracking and follow-up [15]. Hague et al. [16] present an application of a data-to-care approach implemented by the Massachusetts Department of Public Health involving the generation of an out-of-care list of patients lacking a laboratory test result for at least a 6 month period. Swain et al. [17] describe their process in the production of data packages that integrate surveillance and treatment data. The data packages were customized for regional use by twelve health care organizations engaged in the NYLinks initiative. This innovative Data to Care model utilized a multi-phase end-user participatory approach to collaboratively refine these data dissemination products.

The SPNS System's Linkages demonstration was markedly different from previous SPNS initiatives in its adoption of the Collaborative Model developed by the Institute for Healthcare Improvement [18]. Based on its prior experience with Cross-Part initiatives, $\mathrm{HAB}$ required the use of an adaptation of the Model for this initiative to explore how its scope might be expanded beyond its traditional focus on quality improvement to the development and implementation of regional and statewide community-level interventions. DeLorenzo et al. [19] explain how this adoption shaped the overall initiative, describing the mechanics of implementing the Model's Learning Collaborative meetings where stakeholders from all over their respective states worked together to craft the innovative interventions, and in so doing created cooperation and buy-in. As such, this approach shows great promise for creating collaborative partnerships at regional and statewide levels to improve the care and treatment of PLWH.

Implementation science is becoming increasingly important in assuring the successful replication and adaptation of interventions across a range of health issues. Eccles and Mittman define implementation science as "the scientific study of methods to promote the systematic uptake of research findings and other evidence-based practices into routine practice to improve the quality and effectiveness of health services and care" [20]. Best practices may be identified by studying the processes behind the implementation of interventions, including the facilitators and barriers to their successful execution, and strategies to overcome those challenges. Addison et al. describe a mixed-methods implementation science assessment of three interventions implemented by twelve HIV service delivery organizations across New York State in their NYLinks project [21]. In its future demonstration projects, HRSA/HAB intends to emphasize implementation science-driven evaluation efforts to produce robust, replicable interventions that RWHAP recipients may employ in their efforts to comprehensively treat and care for PLWH.

Taken together, these articles demonstrate the importance of the approaches taken in this initiative-to combine datadriven and innovative service models into comprehensive strategies to support linkage and engagement in HIV care for vulnerable populations. These projects hold practical lessons for others interested in enacting efficient, effective models to support PLWH in their jurisdictions in obtaining the best possible health outcomes.

Funding Funding was provided by Health Resources and Services Administration (Grant No: U90HA22702).

\section{References}

1. Office of National AIDS Policy. National HIV/AIDS Strategy for the United States. https://www.hiv.gov/federal-response/nationalhiv-aids-strategy/overview (accessed 22 Nov 17).

2. Office of National AIDS Policy. National HIV/AIDS Strategy Updated to 2020, July 2015. https://www.hiv.gov/federal-respo nse/national-hiv-aids-strategy/nhas-update (accessed 22 Nov 17).

3. Bradford J, Coleman S, Cunningham W. HIV system navigation: an emerging model to improve HIV care access. AIDS Patient Care STDs. 2007;21(S1):S49-58.

4. Schumann CL, Westergaard RP, Meier AE, Ruetten ML, Vergeront JM. Developing a patient navigation program to improve engagement in HIV medical care and viral suppression: a demonstration project protocol. AIDS Behav. 2017. https://doi. org/10.1007/s10461-017-1727-4.

5. Fuller SM, Koester KA, Maiorana A, Steward WT, Broaddus MR, Lass K, Zamudio-Haas S, Quinlivan EB, Myers JJ. I don't have to do this all by myself: systems navigation to ensure continuity of HIV care for persons leaving prison. AIDS Behav. 2018. https:// doi.org/10.1007/s10461-018-2050-4.

6. Bailey S, Gilmore K, Yerkes L, Rhodes A. Connecting corrections and HIV care: building a care coordination program for recently incarcerated persons living with HIV in Virginia. AIDS Behav. 2017. https://doi.org/10.1007/s10461-017-2003-3.

7. Brantley A, Page KM, Zack B, Friedrich KR, Wendell D, Gruber D. Making the connection: using videoconferencing to increase 
linkage to care for incarcerated persons living with HIV postrelease. AIDS Behav. 2018. https://doi.org/10.1007/s1046 1-018-2115-4.

8. CDC. Use of Social networks to identify persons with undiagnosed HIV infection-seven U.S. cities, October 2003-September 2004. Morb Mortal Wkly Rep, 2005; 54 (24): 601-05. http://www.cdc.gov/mmwr/preview/mmwrhtml/mm5424a3.htm (Accessed 24 Oct 17).

9. Schumann C, Kahn D, Broaddus M, Dougherty J, Elderbrook M, Vergeront J, Westergaard R. Implementing a standardized social networks testing strategy in a low HIV prevalence jurisdiction. AIDS Behav. 2018. https://doi.org/10.1007/s10461-018-2146-x.

10. LeViere A, Donovan J, Wilkin A, Keller J, Parnell H, Sampson L, Gay CL, Quinlivan EB. Results of a social network testing intervention for HIV in infectious disease clinics. AIDS Behav. 2018. https://doi.org/10.1007/s10461-018-2178-2.

11. Fadul N, Willis SJ, Donovan J, Wilkin A, Heine AD, LeViere A, Dortche C, Quinlivan E. Characteristics of out-of-care patients who required a referral for re-engagement services by public health bridge counselors following a brief clinic-based retention intervention. AIDS Behav. 2018. https://doi.org/10.1007/s1046 1-018-2110-9.

12. Parnell HE, Berger MB, Gichane MW, LeViere AF, Sullivan KA, Clymore JM, Quinlivan EB. Lost to care and back again: patient and navigator perspectives on HIV care re-engagement. AIDS Behav. 2017. https://doi.org/10.1007/s10461-017-1919-y.

13. CDC. Data to care- using HIV surveillance data to support the HIV Care Continuum. https://effectiveinterventions.cdc.gov/en/ HighImpactPrevention/PublicHealthStrategies/DatatoCare.aspx (accessed 30 May 17).

14. Herwehe J, Wilbright W, Abrams A, Bergson S, Foxhood J, Kaiser M, Smith L, Xiao K, Zapata A, Magnus M. Implementation of an innovative, integrated electronic medical record (EMR) and public health information exchange for HIV/AIDS. J Am Med Inform Assoc. 2011;19(3):448-52.

15. Zamudio-Haas S, Koester KA, Maiorana A, Fuller SM, Steward WT, Gruber D, Hauge JC, Quinlivan EB, Myers JJ. "Closing the loop" developing state-level data sharing interventions to promote optimum outcomes along the HIV Continuum of Care. AIDS Behav. 2018. https://doi.org/10.1007/s10461-018-2142-1.
16. Hague JC, John B, Goldman L, Nagavedu K, Lewis S, Hawrusik R, Rajabiun S, Cocoros N, Fukuda HD, Cranston K. Using HIV surveillance laboratory data to identify out-of-care patients. AIDS Behav. 2017. https://doi.org/10.1007/s10461-017-1742-5.

17. Swain CA, Sawicki S, Addison D, Katz B, Piersanti K, BaimLance A, Gordon D, Anderson BJ, Nash D, Steinbock C, Agins B. An end-user participatory approach to collaboratively refine HIV care data, the New York State experience. AIDS Behav. 2018. https://doi.org/10.1007/s10461-018-2104-7.

18. Institute for Healthcare Improvement. The Breakthrough Series: IHI's collaborative model for achieving breakthrough improvement. IHI innovation series white paper. Boston: Institute for Healthcare Improvement; 2003. http://www.ihi.org/resources/ Pages/IHIWhitePapers/TheBreakthroughSeriesIHIsCollaborat iveModelforAchievingBreakthroughImprovement.aspx (accessed 2 Jun 17).

19. DeLorenzo LA, Fox J, Quinlivan EB, Gilmore KM, Ruetten M, Broaddus M, Steward W, Meyers J. Lessons learned from applying a modified learning collaborative model to promote change in regional and statewide HIV care systems. AIDS Behav. 2018. https://doi.org/10.1007/s10461-018-2176-4.

20. Eccles MP and Mittman BS. Welcome to Implementation Science. Implement Sci, 2006; 1(1): 1-3. https://implementations cience.biomedcentral.com/track/pdf/10.1186/1748-5908-11 ? site $=$ implementationscience.biomedcentral.com (accessed 14 Feb 18).

21. Addison D, Baim-Lance A, Suchman L, Katz B, Swain CA, Piersanti K, Steinbock C, Sawicki S, Agins B, Nash D, on behalf of the NYLinks Implementation Team. Factors influencing the successful implementation of HIV linkage and retention interventions in health care agencies across New York State. AIDS Behav. 2018. https://doi.org/10.1007/s10461-018-2060-2.

Publisher's Note Springer Nature remains neutral with regard to jurisdictional claims in published maps and institutional affiliations. 